\title{
Risk factors for surgical site infection after groin hernia repair: does the mesh or technique matter?
}

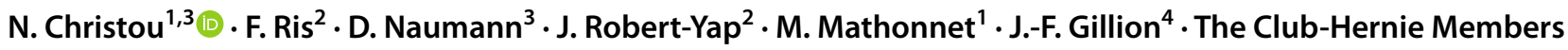

Received: 22 July 2021 / Accepted: 21 September 2021 / Published online: 1 October 2021

(c) The Author(s) 2021

\begin{abstract}
Introduction Surgical site infections (SSIs) following groin hernia repair (GHR) are getting rarer in high income countries despite a wider use of meshes. Among the risk factors for SSIs, those related to the mesh and the surgical technique have rarely been described.

Methods A registry-based multicenter study using prospectively collected data, including SSIs and their potential risk factors, was conducted in the French Hernia-Club.

Results Between 2012 and 2019, 21,976 consecutive unselected adult patients aged $64.8 \pm 15.4$ years old (88.9\% male) underwent GHR (83.5\% unilateral). Fifty four percent were laparoscopic; $97.6 \%$ used mesh. The overall incidence of SSI was $0.26 \%$. The incidence of SSI was respectively, $0.24 \%$ and $0.19 \%(p=0.420)$ in open vs laparoscopic repairs; $0.19 \%$ and $0.25 \%$ ( $p=0.638$ ) for polyester vs polypropylene mesh; In adjusted multivariate analysis focusing on macroporous meshes (which were the most implanted meshes: 23,148 out of 24,099), there were no differences in terms of SSIs' rates regarding the technique: open versus laparoscopy ( $p=0.762)$ nor the type of mesh used: polypropylene versus polyester $(p=0.557)$. Conclusion The rate of SSI following GHR was low in this large registry study. Mesh type and surgical technique did not affect SSIs rates. Caution is advised when interpreting these data due to this very low rate of SSI and the potential for a type II error.
\end{abstract}

Keywords Surgical site infection $\cdot$ Groin hernia repair $\cdot$ Risk factors

\section{Abbreviations \\ GHR Groin hernia repair \\ SSI Surgical site infection}

\section{Introduction}

Groin hernia repair (GHR) is the most frequent intervention in elective general surgery. It represents around 2000 operations per million people per year in High Income Countries [1].

According to systematic reviews [1]2 updated in the Herniasurge guidelines (Herniasurge) [3] the rate of surgical site infections (SSIs) after GHR broadly varies across the studies from $1.6 \%$ in low-risk environment to $4.2 \%$ [4] in high-risk environment. Recently published rates are even lower: $0.4 \%$ in the US NSQIP (National Surgery Quality Improvement Program) [5]; $0.64 \%$ decreasing to $0.2 \%$ after laparoscopic GHR [6].

Numerous risk factors for SSIs after (elective) GHR (such as diabetes, current smoking) have already been identified [5]. Among the risk factors for SSIs, those potentially related to the type of mesh and the surgical technique used have rarely been studied.

3 University Hospitals Birmingham NHS Foundation Trust, Birmingham B152TH, UK

4 Unité de Chirurgie Viscérale Et Digestive, Hôpital Privé d'Antony, 1, Rue Velpeau, 92160 Antony, France 
Hence, the aim of this study, was to focus on type of mesh and surgical technique as potential risk factors for SSI after elective GHR.

\section{Materials and methods}

\section{Study design}

We conducted an observational cohort study using a prospective database (close ended input boxes) of patients who had GHR during a 7-year period from different French centres. This database included all patients over the age of 16 years old who were operated on from GHR in the French Hernia-Club registry between 2012 and 2019. The patients were informed that their anonymous data were registered and that they would receive a phone questionnaire at different stages of their follow-up. Only the operating surgeon and the CRA were able to link the randomly allocated identifying number and the patient. The data were stored in a specialized Swiss data bank where they were protected against network intrusion. The registry complies with the requirements of the General Data Protection Regulation (GDPR), the French 'Méthodologies de référence de la Commission Nationale Informatique et Liberté' (MR001, MR003) and the different specific French ethics committees. STROBE (Strengthening the Reporting of Observational studies in Epidemiology) [5] and the European Registry of Abdominal Wall Hernias (EuraHS) recommendations [6] were used for the conduct and reporting of our study.

\section{Club-Hernie registry}

The French "Club Hernie" is composed of surgeons specially interested in hernia repairs. Hernia club registry is composed of comprehensive anonymous patient data as a registered part of the operating surgeons' office files [not in the online database, to comply with the European General Data Protection Regulation (GDPR)]. All patients' anonymous data are registered in the online database, in real time, consecutively, without selection. The comprehensiveness is ensured with a signed quality charter and checked with audit-reconciliations between the entries in the database and the administrative parietal activity records of the member. Completion gauges, updated with every input, help the surgeon to ensure the comprehensiveness of data.

\section{Patient follow up}

Clinical follow-up was performed by the operating surgeon at discharge and at the first month clinical visit. In case of any symptoms, an additional visit was scheduled between the $3 \mathrm{rd}$ and the 6 th month post-operative check. The followup at 2 years $(2 \mathrm{Y}-\mathrm{Fu})$ and 5 years $(5 \mathrm{Y}-\mathrm{Fu})$ consisted of a telephone interview following a validated phone questionnaire performed by an independent clinical research assistant (CRA) blinded to the surgical procedure. The wording of the questionnaire used layperson terminology and the four level VRS (Verbal Rating Scale). Answers were recorded verbatim, without any medical correction according to our Patient Reported Outcome Measures (PROM) policy. In case of any reported event, the patient was strongly recommended to schedule a clinical visit. A retro-control of the registered outcomes was done during the phone interview. In case of discrepancy, the medical chart was reviewed with the operating surgeon. Patients were considered lost to follow-up after five failed attempts to contact them on different days. Patients who decline to participate in the telephone interview were considered lost to follow-up but also recorded apart as potentially poor outcomes.

\section{Data collection}

Data extracted from the registry included pre-operative data with patient characteristics, factors influencing wound healing or dissection, surgical history and hernia characteristics. Operative characteristics were also described, and included: open or laparoscopic; surgical technique; use of mesh; technique of mesh placement; mesh characteristics; duration of operation; and management of nerve dissection (preservation of nerves, section with buried or coagulated ends). Post-operative outcomes occurring in the first 30 days after the surgery were collected. Complications were graded according to the Clavien-Dindo classification. At every follow-up, a quality of life (QoL) questionnaire and a patient self-assessment of the surgery (PROM) form were filled out and registered in the database.

\section{Outcomes}

The primary outcome measure was the occurrence of any SSI within 1 year of postoperative follow-up. These infections were detected at regular clinical controls at discharge, at the 1-month post-operative clinical visits and during any additional visits in case of any symptoms during the 1-year postoperative period. SSI was defined following the Centers for Disease Control and Prevention criteria [7]. SSIs encompassed superficial, deep, and organ space infections. More 
precisely, we included patients with either non peri-mesh infected collection (subcutaneous) or peri-mesh infected collection. We also recorded whether SSI was managed with mesh conservation or with mesh explant.

\section{Data analysis}

Categorical data are summarized as number and percentage, and normal continuous data are summarized as mean and standard deviation $( \pm$ SD). Comparisons were conducted using Chi-square tests (or Fisher exact tests if the conditions to apply Chi-square were not verified by data). The difference in risk of SSI between polyester and polypropylene prosthesis was assessed and reported with an exact $95 \%$ confidence interval (95\% CI). Adjusted associations with the risk of SSI were investigated with a multiple logistic regression model. Due to the low number of SSIs, the number of independent variables was limited to five. These independent variables were selected a priori and no statistical procedure of variable selection was used. Unadjusted and adjusted odds ratios were reported with their $95 \%$ confidence intervals. The type I error was 0.05 two-sided for all statistical analyses. Analyses were conducted with software R version 4.0.2 (Foundation for Statistical Computing, Vienna, Austria) using the R package Desctools [Andri Signorell et mult. al. (2020). DescTools: tools for descriptive statistics, $\mathrm{R}$ package version 0.99.38].

\section{Results}

\section{Patients' characteristics}

During the study period (June 2011-May 2019), 25,499 patients underwent GHR in elective surgery conditions. 86 surgeons participated in this study. After exclusion of patients with missing data, there were 21,976 patients who had 25,593 GHR (accounting for patients who had bilateral surgery). The mean age was 64.8 years old $( \pm 15.4)$ years old, the majority of whom were male (88.9\%). Of the 25,593 groin hernias, 24,076 were repaired with mesh and 589 without.

\section{Surgical site infections}

Surgical site infections (SSIs) were identified in 57 patients $(0.26 \%)$ within the study cohort. The groups with and without SSIs were comparable in terms of demographic characteristics (Table 1a, b) and surgical characteristics (Table 2a). There was no statistically significant difference between the two groups with SSIs or without, regarding the operated side (left or right), the operated hernia recurrent or primary, the technique used (laparoscopic or laparotomy and with or without a mesh), the management of nerves or the use of naropeine (ropivacaine) infiltration (Table 2a). Similarly, there was no significant difference between the two groups with or without SSI when a mesh was implanted regardless of the surgical characteristics already described above, the mesh characteristics, or the type of fixation, Table $2 b$.

\section{Risk factors for SSI}

\section{Univariate analysis}

Results of univariate analysis are shown in Tables 3. Neither factors linked to the patients' characteristics nor factors linked to wound healing (Table $3 \mathrm{a}$ ) were independently associated with SSI. Considering surgical factors (Table $3 b$ ), none were independently associated with SSI. Furthermore, focusing on sides with a mesh, no factors linked to surgery such as recurrences, technique used (open or laparoscopy), or mesh characteristics were associated with SSI occurrence ( $p=0.638$ for mesh type and $p=0.406$ for mesh porosity) (Table S1). Noticeably, no statistically significant difference in the SSI rate was observed between macroporous polyester vs polypropylene GHR (Table 3c). More precisely, the difference between polyester and polypropylene (both non-resorbable prostheses) was $-0.06 \%(-0.18-0.06,95 \%$ CI) $(p=0.395)$ (Table 3c). The method of fixation of the mesh, nerve preservation and naropeine infiltration were not significantly linked to higher SSI rates (Table S1).

\section{Multivariate analysis}

Regardless of the association between technique used (laparoscopy or open) and the type of hernia operated (uni or bilateral), there was no significant difference in SSI number $(p=0.267)$ After logistic regression adjusted for ASA score, bilateral hernia surgery or open surgery, none of these factors were linked to an increased risk of SSI (Table 4a). Further multivariate analysis was performed on the macroporous mesh group (number of sides analyzed $=23,141$ ). After adjustment of BMI, smoking status, ropivacaine infiltration and surgical technique used, there was no difference in SSI risk between each type of macroporous mesh used, especially between polyester and polypropylene meshes ( $p$ $=0.557)$ (Table 4b).

\section{Discussion}

We report an overall $0.26 \%$ rate of SSI (by patient and by operated side) for a cohort of 21,976 patients having undergone an elective GHR and registered in the French Hernia club registry. They were considered as elective procedures (24,076 sides with a mesh and 589 sides without mesh), 
Table 1 Patients' characteristics

\begin{tabular}{|c|c|c|c|}
\hline & All patients $(n=21,976)$ & No SSI $(n=21,919)$ & $\operatorname{SSI}(n=57)$ \\
\hline Bilateral & $3617(16.5 \%)$ & $3607(16.5 \%)$ & $10(17.5 \%)$ \\
\hline Unilateral & $18,359(83.5 \%)$ & $18,312(83.5 \%)$ & $47(82.5 \%)$ \\
\hline \multicolumn{4}{|l|}{ Gender } \\
\hline Female & $2446(11.1 \%)$ & $2440(11.1 \%)$ & $6(10.5 \%)$ \\
\hline Male & $19,530(88.9 \%)$ & $19,479(88.9 \%)$ & $51(89.5 \%)$ \\
\hline Age, years & $64.8(15.4)$ & $64.8(15.4)$ & $63.5(16.8)$ \\
\hline BMI & $24.9(3.4)$ & $24.9(3.4)$ & $25.5(3.2)$ \\
\hline \multicolumn{4}{|l|}{ ASA score } \\
\hline I & $10,609(49 \%)$ & $10,583(49 \%)$ & $26(48.1 \%)$ \\
\hline II & $8049(37.2 \%)$ & $8026(37.1 \%)$ & $23(42.6 \%)$ \\
\hline III & $2962(13.7 \%)$ & $2957(13.7 \%)$ & $5(9.3 \%)$ \\
\hline IV & $39(0.2 \%)$ & $39(0.2 \%)$ & $0(0 \%)$ \\
\hline $\mathrm{V}$ & $2(0 \%)$ & $2(0 \%)$ & $0(0 \%)$ \\
\hline Abdominal pressure & $5964(28.1 \%)$ & $5950(28.1 \%)$ & $14(25.5 \%)$ \\
\hline Smoking & $3213(15.2 \%)$ & $3206(15.2 \%)$ & $7(13.2 \%)$ \\
\hline Physical effort & $7130(34.2 \%)$ & $7114(34.2 \%)$ & $16(28.6 \%)$ \\
\hline$\underline{\text { Dissection factors }}$ & $6791(32.2 \%)$ & $6777(32.3 \%)$ & $14(26.4 \%)$ \\
\hline \multicolumn{4}{|c|}{ b: Patients' characteristics—wound healing factors } \\
\hline & All patients $(n=21,976)$ & No SSI $(n=21,919)$ & $\operatorname{SSI}(n=57)$ \\
\hline Diabetes & $1069(4.3 \%)$ & $1066(4.3 \%)$ & $3(5.5 \%)$ \\
\hline Corticotherapy & & $325(1.3 \%)$ & $323(1.3 \%)$ \\
\hline Pelvic radiotherapy & $305(1.2 \%)$ & $305(1.2 \%)$ & $0(0.0 \%)$ \\
\hline Chemotherapy & $0(0.0 \%)$ & $0(0.0 \%)$ & $0(0.0 \%)$ \\
\hline Anticoagulants & $3339(13.6 \%)$ & $3331(13.6 \%)$ & $8(14.5 \%)$ \\
\hline
\end{tabular}

and classified as "clean surgery" without any bowel contamination (Altemeier Class 1). To our knowledge, this is the largest study of elective groin hernia repairs that investigates post-operative infection rates and SSIs risk factors potentially related to both mesh and surgical technique used including both laparoscopic and open procedures. Indeed, a recent study only explored the demographics and the preoperative risk factors (and encompassed emergency GHR) [6], another one compared, as second endpoint, the incidence of SSIs between laparoscopic and Lichtenstein techniques within a male cohort only [8].

Interestingly, in our study, after adjusted multivariate analysis, we didn't highlight any risk factors of SSIs regarding both mesh type and technique (open versus laparoscopy) used.

The main advantage of our large French cohort, was that we were able to investigate which mesh characteristics might impact on SSI rates. Our work demonstrated no specific mesh features impact on SSI risk. No statistically significant difference in the SSI rate was observed between macroporous polyester vs polypropylene GHR, just a trend favouring polyester after adjusted multivariate analysis (Table 4b).
The recent cohort of 54,951 cases from the American College of Surgery National Surgical Quality Improvement Program (ACS NSQIP) reported by Sereysky et al. [5] focused on elective open repairs only. No risk factors for SSIs were found in both univariate and multivariate analyses of 18 parameters related to patients, surgery, or operative characteristics. These divergences of results from Sereysky et al. with our study, may be explained by the differences regarding (i) the methodology: only open repairs were included in the cohort of Sereysky et al. whereas we integrated open and laparoscopic repairs in our study, (ii) the characteristics of the cohort: percentage of patients within the American cohort with general risk factors of infections such as type 2 diabetes, obesity, current smoking, use of steroids, was higher.

In another large study on 17,388 patients from the Herniamed database [8], the SSI rates were statistically different $(0.06 \%$ vs. $0.26 \% ; p=0.003)$ in total extra peritoneal repair (TEP repair- by laparoscopy) and Lichtenstein technique (open). In our present study, the incidence of SSI ( $0.19 \%$ vs $0.24 \% ; p=0.486$ ) was not significantly different in the open vs. laparoscopic group. This difference may be lightened by 
Table 2 Surgical characteristics

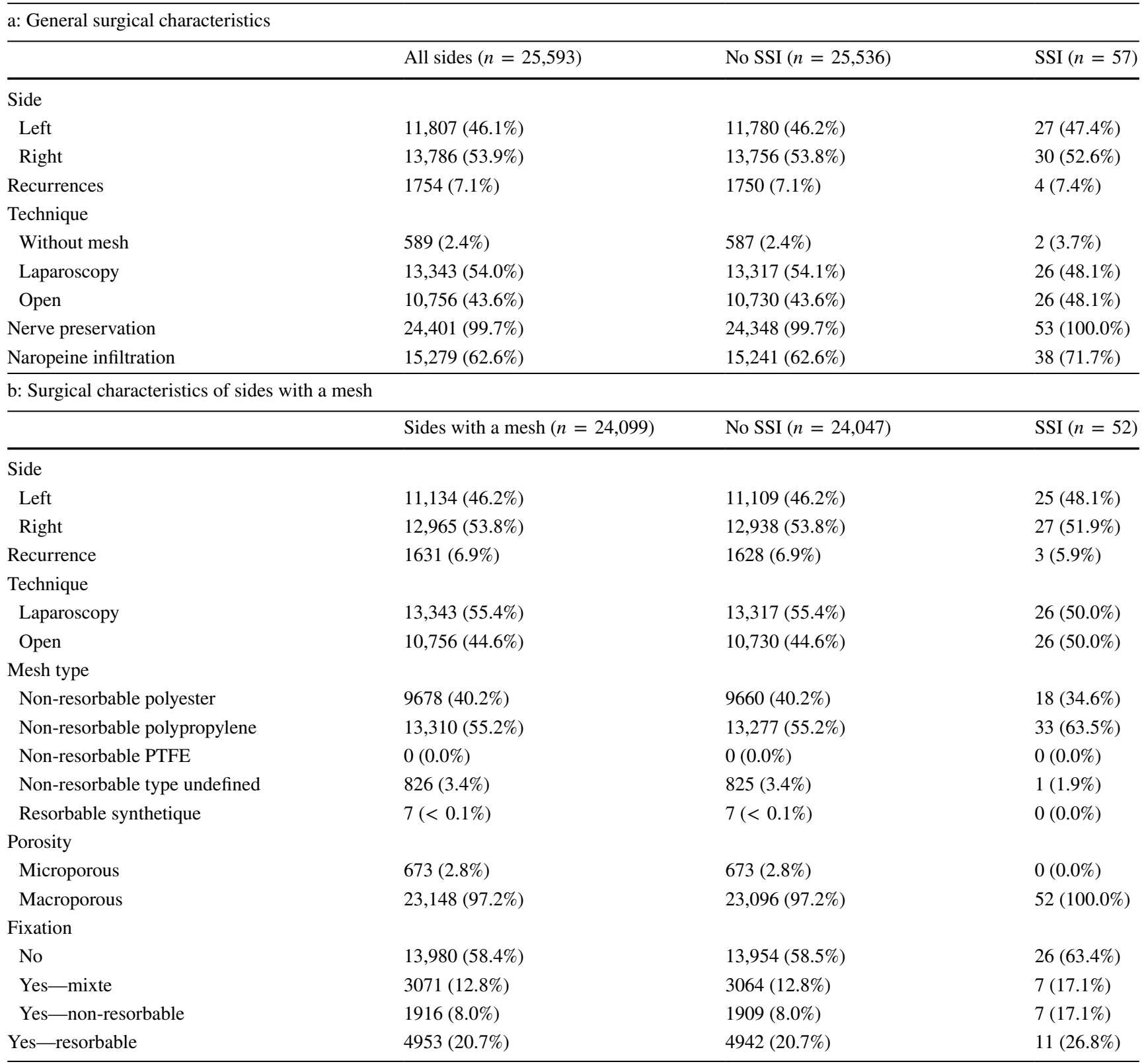

the fact that in the French Hernia Club registry, Lichtenstein procedures are performed by specialists of this technique.

From an international point of view, ours is the first study to our knowledge to investigate the number of SSIs in elective surgery (without emergencies [9]). Previous data that examined SSI following elective GHR were derived from investigation of the merits of antibiotic prophylaxis [10]. SSI rates after GHR were reported as between 2.4 and $6 \%$, which is higher than the current data. This may be explained by the fact that our database grouped high volume centres (around 150 GHR per year per surgeon). In France, since 2014, the French National monitoring program of surgical site infections (SSI) (called ISO-RAISIN) [11] has shown a steady and significant increase of the number of SSIs after GHR: $0.67 \%$ in 2013 versus $0.93 \%$ in 2017 . These figures are threefold higher than our results. This difference may be explained once again by the fact that the database of "Club Hernie" comes from high-volume centres and surgeons, while the database of ISO-RAISIN only collects data from voluntary medical centres. This may favour highly specialized centres for groin hernia surgery as has previously been demonstrated in different studies worldwide [12]1314. Moreover, in the French report, the ISO-RAISIN data are recorded for both "groin" and "ventral" hernias which are completely different from only "groin hernias" which have different risk factors for SSIs. 
Table 3 Univariate analysis - risk factors of SSIs: patients and surgical factors

a: Patient factors ( $n=21,976$ patients)

\begin{tabular}{|c|c|}
\hline & Risk of SSI \\
\hline Bilateral & $p=0.966$ \\
\hline Bilateral & $10 / 3617(0.28 \%)$ \\
\hline Unilateral & $47 / 18359(0.26 \%)$ \\
\hline Gender & $p>0.99$ \\
\hline Women & $6 / 2446(0.25 \%)$ \\
\hline Men & $51 / 19530(0.26 \%)$ \\
\hline Age, years & $p=0.305$ \\
\hline $16-54$ & $16 / 5308(0.30 \%)$ \\
\hline $55-64$ & $7 / 4563(0.15 \%)$ \\
\hline $65-74$ & $14 / 6011(0.23 \%)$ \\
\hline 75 -and + & 20/6094 (0.33\%) \\
\hline BMI & $p=0.666$ \\
\hline Less than 20 & $2 / 1147(0.17 \%)$ \\
\hline $20-25$ & $25 / 10805(0.23 \%)$ \\
\hline $26-30$ & $25 / 8317(0.30 \%)$ \\
\hline 31 and + & $5 / 1454(0.34 \%)$ \\
\hline ASA score & $p=0.531$ \\
\hline I & $26 / 10609(0.25 \%)$ \\
\hline II & $23 / 8049(0.29 \%)$ \\
\hline III-V & $5 / 3003(0.17 \%)$ \\
\hline Abdominal pressure & $p=0.771$ \\
\hline No & $41 / 15241(0.27 \%)$ \\
\hline Yes & $14 / 5964(0.23 \%)$ \\
\hline Smoking & $p=0.834$ \\
\hline No & $46 / 17953(0.26 \%)$ \\
\hline Yes & $7 / 3213(0.22 \%)$ \\
\hline Physical effort & $p=0.456$ \\
\hline No & $40 / 13730(0.29 \%)$ \\
\hline Yes & $16 / 7130(0.22 \%)$ \\
\hline Wound healing factors & $p=0.774$ \\
\hline No & $42 / 16605(0.25 \%)$ \\
\hline Yes & $13 / 4454(0.29 \%)$ \\
\hline \multicolumn{2}{|c|}{ b: Surgical factors ( $n=25,593$ sides $)$} \\
\hline & Risk of SSI \\
\hline Recurrences & $p=0.792$ \\
\hline No & $50 / 22988(0.22 \%)$ \\
\hline Yes & $4 / 1754(0.23 \%)$ \\
\hline Technique & $p=0.420$ \\
\hline No mesh & $2 / 589(0.33 \%)$ \\
\hline Laparoscopy & $26 / 13343(0.19 \%)$ \\
\hline Open & $26 / 10756(0.24 \%)$ \\
\hline Dissection factors & $p=0.446$ \\
\hline No & $39 / 14271(0.27 \%)$ \\
\hline Yes & $14 / 6791(0.21 \%)$ \\
\hline Nerve preservation & $p>0.99$ \\
\hline No & $0 / 75(0.00 \%)$ \\
\hline Yes & $53 / 24401(0.22 \%)$ \\
\hline
\end{tabular}


Table 3 (continued)

\begin{tabular}{lr}
\hline b: Surgical factors $(n=25,593$ sides $)$ & Risk of SSI \\
\hline & $p=0.219$ \\
\hline Naropeine infiltration & $15 / 9128(0.16 \%)$ \\
No & $38 / 15279(0.25 \%)$ \\
Yes & $p=0.957$ \\
Side & $27 / 11807(0.23 \%)$ \\
Left & $30 / 13786(0.22 \%)$ \\
Right & 3
\end{tabular}

Table 4 Multivariate analysis

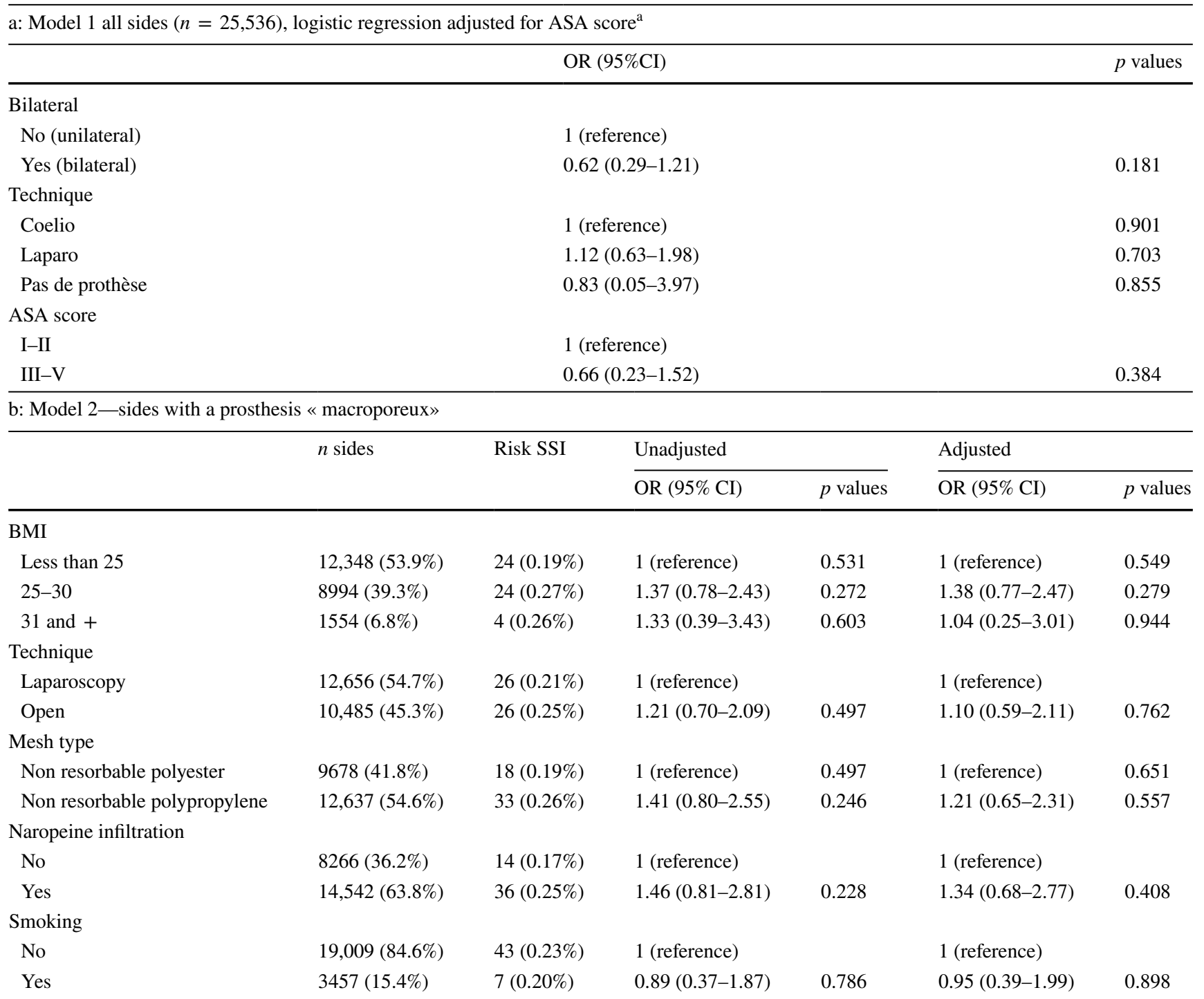

${ }^{\mathrm{a}}$ Interaction term between bilateral and technique was not statistically significant $(p=0.388)$

Investigations of risk factors for SSI following GHR in the literature are sparse, and usually concentrates on patientrelated predictive factors only. The study by Taylor et al. [15] in 2004 included data from 32 Scottish hospitals with
2,665 patients who had groin hernia surgery. Overall, 5.2\% had SSI. The study pointed out after two multivariate analyses, two SSI risk factors: absence of antibiotic prophylaxis $(p=0.002)$ and an NNIS (National nosocomial infections 
surveillance system) local score of 1 or $2(p=0.021)$. The NNIS score is calculated by assigning one point to each of the following risk factors present: ASA score of 3 or more, operation classified as contaminated or dirty, and operation lasting less than $55 \mathrm{~min}$, On the other hand, the study by Sereysky et al. [5], collected all the data of the American College of Surgeons National Surgical Quality Improvement Program (NSQIP). It included patients older than 18 years old with elective initial open inguinal hernia repair (thus excluding bilateral hernias, emergencies, unclean surgeries and operations conducted by laparoscopy). They reported SSI for $0.4 \%$ patients. After multiple logistic regression, only 3 factors (out of 17) were independently associated with SSIs: diabetes, BMI $\geq 35 \mathrm{~kg} / \mathrm{m}^{2}$, and current smoking. In the latter study, antibiotic prophylaxis was not tracked. According to the HerniaSurg group, guidelines published in 2018, antibiotic prophylaxis is recommended in "open mesh repair in a high-risk environment" [3]. In our study, antibiotic prophylaxis was not used according to the previous guidelines and those of the French association of Anaesthesia (SFAR) until 2018. However, in 2018, SFAR guidelines changed and recommended antibiotic prophylaxis for mesh repair whatever the surgical approach. We can consider that between June 2018 (date of the 2018 SFAR guidelines) and May 2019 (end of our study), antibiotic prophylaxis in our study was used when recommended. Despite that, it seems evident that no difference exists between the two groups having benefited from a mesh repair whatever the surgical approach: or whether antibiotic prophylaxis was given. Furthermore, our study is different with $54 \%$ of patients operated laparoscopically and $16.5 \%$ with bilateral hernia repair. Our study is the only one to demonstrate the absence of difference in terms of SSI rates between open GHR and laparoscopic GHR, especially without antibiotic prophylaxis. This result is indirectly in accordance with the one of Köckerling et al. [6] where the benefit of antibiotics could only be limited at the open GHR because in fact they integrated emergency GHR in their analysis.

Concerning fixation method, our results seem similar to those of the review of Sanders et al. [16]. However, it is important to underline that this review encompassed twelve randomized clinical trials (majority of which were assessed as low or very low quality), including only 1992 primary open anterior inguinal hernia repairs. Four studies compared n-butyl-2 cyanoacrylate (NB2C) glues to sutures, two compared self-fixing meshes to sutures, four compared fibrin sealant to sutures, one compared tacks to sutures, and one compared absorbable sutures to non-absorbable sutures. No significant difference in surgical site infection rates between fixation methods were demonstrated.

Köckerling et al. [17] analyzed patients with inguinal hernia repair from the Herniamed registry. Those patients taking anticoagulants were significantly associated with higher deep infection rates, albeit for incisional hernias rather than GHR. In our study, that focused on GHR, treatment with anticoagulants was not associated with higher SSI rates.

\section{Limitations}

The main limits of our study are respectively the involvement of French and high volumes centres only.

Moreover, the absence of significance regarding the type of mesh used and the risk of SSI, can be explained by the few numbers of SSIs: 57, despite a large number of patients in our cohort, more than 20,000. More precisely, due to the very low incidence of SSI $(0.26 \%)$, despite the large number of cases in this series $(21,976)$, the risk of a type II error must be considered. Indeed, for an exposure with an assumed prevalence of $50 \%$ in a sample, the statistical power was $80 \%$ to detect a risk ratio of 2.2 (corresponding to an incidence of SSI equal to $0.16 \%$ in unexposed persons and $0.36 \%$ in exposed persons). Although the lack of identification of risk factors for SSI could be due to limited statistical power, our cohort is one of the largest reported, which may suggest that a larger sample size would simply identify factors with weak association with SSI, and likely relatively low clinical relevance.

\section{Conclusion}

In our large observational multicentre cohort of 25,593 groin hernia repairs in France, we did not find any patient or surgical risk factors that influenced the risk of SSI within a year of surgery. In particular there were no significant differences in SSI between laparoscopic and open surgery, or between different types of mesh. The overall rate of SSI was very low in our study that included patients from high volume centres, which suggests that the expected SSI rate in such a context is expected to be low.

\section{Appendix 1}

\section{Definitions}

*Mesh characteristics

- Were defined according to Amid's classification [8]: macroporous (prolene/polypropylene), microporous (ePTFE).

- The description of meshes as in «polyester», «polypropylene» «PTFE» corresponds to the main component of the mesh.

More precisely, here, in our study 
Non resorbable polyester and polypropylene meshes were considered as «macroporous».

Non resorbable PTFE meshes were considered as «microporous».

Physical effort encompassed physical work and/or frequent sport (more than once per week).

Dissection factors encompassed surgical history of

- Mac Burney.

- Other intraperitoneal surgery.

- Peritoneal vascular bypass.

- Transvesical adenomectomy.

- Homolateral iliac dissection.

- Radical prostatectomy.

- Other extra peritoneal surgery.

- Pelvic radiotherapy.

Recurrence: clinical or radiological presence of the same groin hernia (on the same side).

Smoking encompassed only daily smokers.

*Hernia characteristics

- Symptomatology of hernia: asymptomatic or symptomatic (with or without emergency criteria).

- Type of hernia: inguinal (direct or indirect), or femoral.

- Primary or recurrent.

- Uni or bilateral.

\section{Appendix 2}

Centers for Disease Control criteria for definition of superficial surgical site infection.

Infection occurs within 30 days after operation and Infection involves only skin or subcutaneous tissue of the incision and at least one of the following

1. Purulent drainage, with or without laboratory confirmation from the superficial incision.

2. Organisms obtained from an aseptically obtained culture of fluid or tissue from the superficial incision.

3. At least one of the following signs or symptoms of infection: pain or tenderness, localized swelling, redness or heat and superficial incision is deliberately opened by surgeon, unless incision is culture negative.

4. Diagnosis of superficial incisional SSI by the surgeon or attending physician.

Do not report the following conditions as SSI

1. Stitch abscess (minimal inflammation and discharge confined to the points of suture penetration).

2. Infection of an episiotomy or newborn circumcision site.
3. Infected burn wound.

4. Incisional SSI that extends into the fascial and muscle layers.

Abdominal pressure

Dysuria ( $>2 /$ night or weak stream or delay before urination).

Constipation $>3$ days.

Strength work (multi daily carrying of loads $>10 \mathrm{kgs}$ ).

Intensive sports activities.

Ascite.

Physical effort

Physical work and/or frequent sport (more than once per week).

Dissection factors: surgical history of

- Mac Burney.

- Other intraperitoneal surgery.

- Peritoneal vascular bypass.

- Transvesical adenomectomy.

- Homolateral iliac dissection.

- Radical prostatectomy.

- Other extra peritoneal surgery.

- Pelvic radiotherapy.

Supplementary Information The online version contains supplementary material available at https://doi.org/10.1007/s10029-021-02512-7.

Funding No funding.

\section{Declarations}

Conflict of interest The authors declare no conflicts of interest. The ICMJE disclosure form has been filled by each author.

Ethical standards The data were stored in a specialized Swiss data bank where they were protected against network intrusion. The registry complies with the requirements of the General Data Protection Regulation (GDPR), the French 'Méthodologies de référence de la Commission Nationale Informatique et Liberté' (MR001, MR003) and the different specific French ethics committees.

Human and animal rights This article does not contain any studies with animals performed by any of the authors.

Informed consent approval The patients were informed that their anonymous data were registered and that they would receive a phone questionnaire at different stages of their follow-up. Only the operating surgeon and the CRA were able to link the randomly allocated identifying number and the patient. 
Open Access This article is licensed under a Creative Commons Attribution 4.0 International License, which permits use, sharing, adaptation, distribution and reproduction in any medium or format, as long as you give appropriate credit to the original author(s) and the source, provide a link to the Creative Commons licence, and indicate if changes were made. The images or other third party material in this article are included in the article's Creative Commons licence, unless indicated otherwise in a credit line to the material. If material is not included in the article's Creative Commons licence and your intended use is not permitted by statutory regulation or exceeds the permitted use, you will need to obtain permission directly from the copyright holder. To view a copy of this licence, visit http://creativecommons.org/licenses/by/4.0/.

\section{References}

1. Sanchez-Manuel FJ, Lozano-García J, Seco-Gil JL (2012) Antibiotic prophylaxis for hernia repair. Cochrane Database Syst Rev 2:CD003769

2. Weyhe D, Tabriz N, Sahlmann B, Uslar V-N (2017) Risk factors for perioperative complications in inguinal hernia repair-a systematic review. Innov Surg Sci 2(2):47-52

3. HerniaSurge Group (2018) International guidelines for groin hernia management. Hernia J Hernias Abdom Wall Surg 22(1):1-165

4. Pande T, Naidu CS (2020) Mesh infection in cases of polypropylene mesh hernioplasty. Hernia J Hernias Abdom Wall Surg 24(4):849-856

5. Sereysky J, Parsikia A, Stone ME, Castaldi M, McNelis J (2020) Predictive factors for the development of surgical site infection in adults undergoing initial open inguinal hernia repair. Hernia 24(1):173-178

6. Köckerling F, Bittner R, Jacob D, Schug-Pass C, Laurenz C, Adolf $\mathrm{D}$ et al (2015) Do we need antibiotic prophylaxis in endoscopic inguinal hernia repair? Results of the Herniamed Registry. Surg Endosc déc 29(12):3741-3749

7. Horan TC, Gaynes RP, Martone WJ, Jarvis WR, Emori TG (1992) CDC definitions of nosocomial surgical site infections, 1992: a modification of CDC definitions of surgical wound infections. Infect Control Hosp Epidemiol 13(10):606-608

8. Köckerling F, Stechemesser B, Hukauf M, Kuthe A, Schug-Pass C (2016) TEP versus Lichtenstein: which technique is better for the repair of primary unilateral inguinal hernias in men? Surg Endosc 30(8):3304-3313

9. GlobalSurg Collaborative (2018) Surgical site infection after gastrointestinal surgery in high-income, middle-income, and lowincome countries: a prospective, international, multicentre cohort study. Lancet Infect Dis 18(5):516-525

10. Boonchan T, Wilasrusmee C, McEvoy M, Attia J, Thakkinstian A (2017) Network meta-analysis of antibiotic prophylaxis for prevention of surgical-site infection after groin hernia surgery. BJS Br J Surg 104(2):e106-e117

11. Astagneau P, L'Hériteau F, Daniel F, Parneix P, Venier A-G, Malavaud $S$ et al (2009) Reducing surgical site infection incidence through a network: results from the French ISO-RAISIN surveillance system. J Hosp Infect 72(2):127-134

12. Aquina CT, Probst CP, Kelly KN, Iannuzzi JC, Noyes K, Fleming FJ et al (2015) The pitfalls of inguinal herniorrhaphy: surgeon volume matters. Surgery 158(3):736-746

13. Andresen K, Friis-Andersen H, Rosenberg J (2016) Laparoscopic repair of primary inguinal hernia performed in public hospitals or low-volume centers have increased risk of reoperation for recurrence. Surg Innov 23(2):142-147

14. Sanders DL, Kingsnorth AN, Windsor ACJ (2016) Is there a role for hernia subspecialists? Or is this a step too far? Hernia 20(5):637-640

15. Taylor EW, Duffy K, Lee K, Hill R, Noone A, Macintyre I et al (2004) Surgical site infection after groin hernia repair. BJS 91(1):105-111

16. Sanders DL, Waydia S (2014) A systematic review of randomised control trials assessing mesh fixation in open inguinal hernia repair. Hernia 18(2):165-176

17. Köckerling F, Roessing C, Adolf D, Schug-Pass C, Jacob D (2016) Has endoscopic (TEP, TAPP) or open inguinal hernia repair a higher risk of bleeding in patients with coagulopathy or antithrombotic therapy? Data from the Herniamed Registry. Surg Endosc 30(5):2073-2081

Publisher's Note Springer Nature remains neutral with regard to jurisdictional claims in published maps and institutional affiliations. 